\title{
Impactos da pandemia da COVID-19 no diagnóstico, atendimento e mortalidade de pacientes oncológicos no Brasil: uma revisão de literatura
}

Impacts of the COVID-19 pandemic on the diagnosis, care and mortality of oncological patients in Brazil: a literature review

Impactos de la pandemia COVID-19 en el diagnóstico, atención y mortalidad de pacientes oncológicos en Brasil: revisión de la literatura

Recebido: 29/09/2021 | Revisado: 04/10/2021 | Aceito: 13/10/2021 | Publicado: 16/10/2021

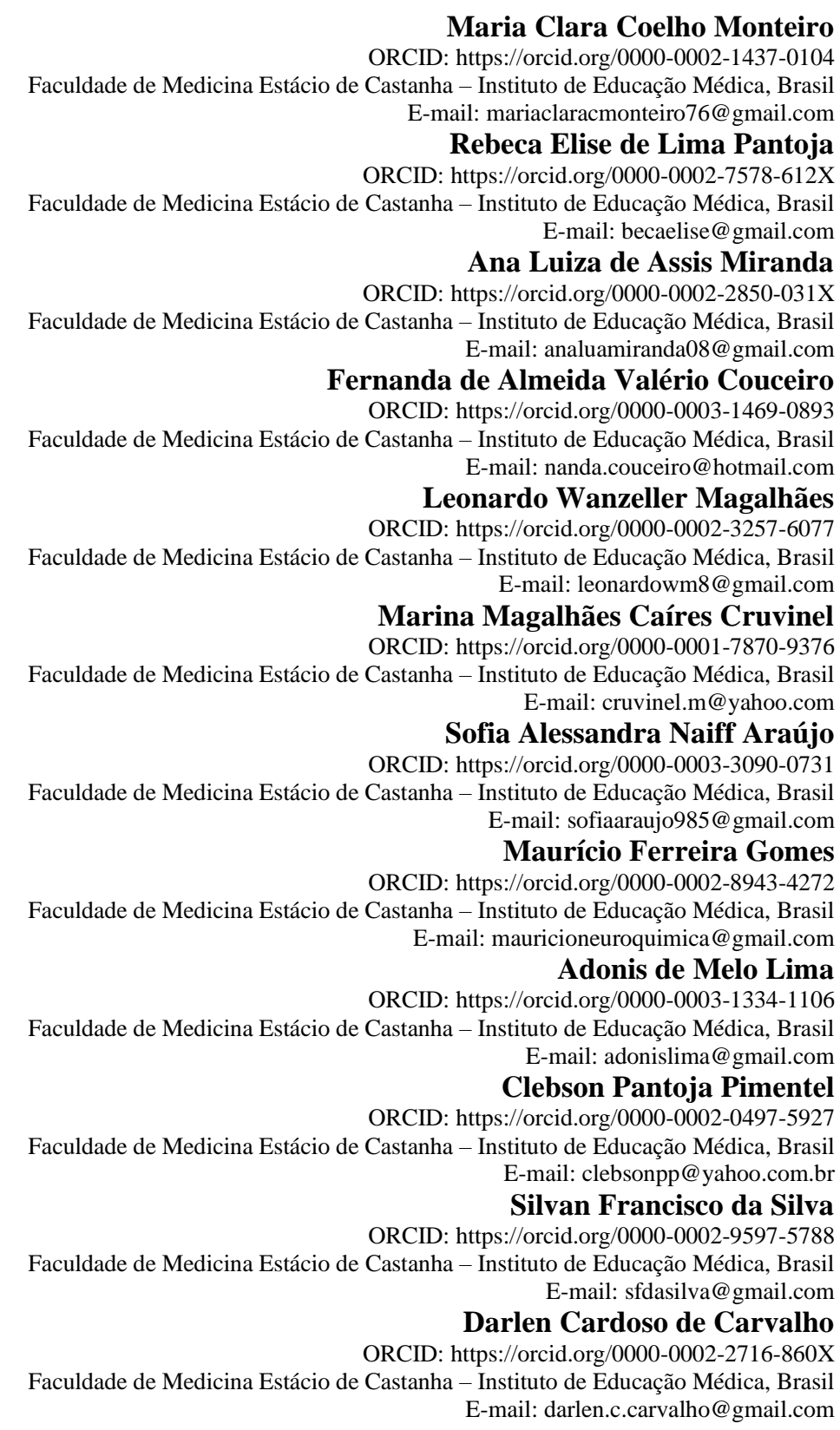

\section{Resumo}

A pandemia COVID-19 afetou gravemente os sistemas de saúde em todo o mundo, afetando a assistência para uma variedade de doenças crônicas, incluindo o diagnóstico e tratamento do câncer. Os pacientes com câncer estão incluídos 
no grupo de vulnerabilidade à maior gravidade e mortalidade por infecção por Sars-CoV-2. Os estudos sobre o impacto do COVID-19 em pacientes brasileiros com câncer são insuficientes na literatura. Este estudo tem como objetivo compilar informações sobre o impacto da pandemia COVID-19 em pacientes brasileiros oncológicos, principalmente sobre diagnóstico, atendimento e mortalidade. Foi realizada uma revisão da literatura nas plataformas Google Scholar, Scielo e Pubmed. Os seguintes descritores foram estabelecidos: "COVID-19", "Câncer", "Mortalidade", "Diagnóstico" e "Atendimento". Um total de 13 artigos foram incluídos na revisão. Os estudos analisados envolveram populações de diferentes regiões brasileiras, principalmente da região Sudeste. Vários tipos de câncer foram observados, com destaque para as neoplasias de cabeça e pescoço. Todos os estudos mostraram redução no número de diagnósticos de câncer, evidenciou-se também déficit no número de consultas, internações e procedimentos cirúrgicos, além de altas taxas de mortalidade para pacientes oncológicos infectados pelo Sars-CoV-2. Em conclusão, a pesquisa demonstrou o enorme impacto do curso da pandemia em pacientes com câncer, ao diminuir o número de diagnósticos, consultas, cirurgias e um aumento significativo na taxa de mortalidade hospitalar. Esses achados indicam as consequências negativas iminentes no diagnóstico e na mortalidade por câncer nos próximos anos e podem alertar para o desenvolvimento de protocolos de saúde e pesquisas contínuas que minimizem esses impactos.

Palavras-chave: COVID-19; Câncer; Diagnóstico; Atendimento; Mortalidade.

\begin{abstract}
The COVID-19 pandemic has severely affected healthcare systems around the world, affecting the assistance for a variety of chronic diseases, including cancer diagnosis and treatment. Cancer patients are included in the group of vulnerability to higher severity and mortality from Sars-CoV-2 infection. Studies on the impact of COVID-19 on Brazilian cancer patients are insufficient in the literature. This study aims to compile information on the impact of the COVID-19 pandemic on Brazilian cancer patients, mainly about diagnosis, care, and mortality. A literature review was carried in Google Scholar, Scielo and Pubmed platforms. The following descriptors were stablished: "COVID-19", "Cancer", "Mortality", "Diagnosis" and "Service". A total of 13 articles were included in the review. The studies analyzed involved populations from different Brazilian regions, mostly in the Southeast region. Multiple types of cancer were observed, with emphasis on head and neck neoplasms. All studies showed a reduction in the numbers of cancer diagnoses, a deficit in the number of appointments, hospitalizations and surgical procedures was also evidenced, in addition to high mortality rates for cancer patients infected by the Sars-CoV-2. In conclusion, the research demonstrated the huge impact of pandemic course on cancer patients by decreasing the number of diagnoses, appointments, surgeries, and a significant increase in the hospital mortality rate. These findings indicate the imminent negative consequences in the diagnosis and mortality from cancer in the following years and could provide warning for the development of health protocols and continuous research that minimize these impacts.
\end{abstract}

Keywords: COVID-19; Cancer; Diagnosis; Service; Mortality.

\title{
Resumen
}

La pandemia de COVID-19 ha afectado gravemente a los sistemas de salud de todo el mundo, afectando la atención de una variedad de enfermedades crónicas, incluido el diagnóstico y el tratamiento del cáncer. Los pacientes con cáncer se incluyen en el grupo de vulnerabilidad a una mayor gravedad y mortalidad por infección por Sars-CoV-2. Los estudios sobre el impacto del COVID-19 en pacientes brasileños con cáncer son insuficientes en la literatura. Este estudio tiene como objetivo recopilar información sobre el impacto de la pandemia COVID-19 en los pacientes brasileños de cáncer, principalmente en el diagnóstico, la atención y la mortalidad. Se realizó una revisión de la literatura en las plataformas Google Scholar, Scielo y Pubmed. Se establecieron los siguientes descriptores: "COVID-19”, "Cáncer", "Mortalidad”, "Diagnóstico" y "Servicio". Se incluyeron un total de 13 artículos en la revisión. Los estudios analizados involucraron poblaciones de diferentes regiones brasileñas, principalmente de la región Sudeste. Se observaron varios tipos de cáncer, especialmente neoplasias de cabeza y cuello. Todos los estudios mostraron una reducción en el número de diagnósticos de cáncer, también se evidenció un déficit en el número de consultas, hospitalizaciones y procedimientos quirúrgicos, además de altas tasas de mortalidad para los pacientes oncológicos infectados con Sars-CoV-2. En conclusión, la investigación demostró el enorme impacto del curso de la pandemia en los pacientes con cáncer, al disminuir el número de diagnósticos, consultas, cirugías y un aumento significativo de la tasa de mortalidad hospitalaria. Estos hallazgos indican las inminentes consecuencias negativas en el diagnóstico y la mortalidad por cáncer en los próximos años y pueden alertar sobre el desarrollo de protocolos de salud e investigación continua que minimicen estos impactos.

Palabras clave: COVID-19; Cáncer; Diagnóstico; Servicio; Mortalidad.

\section{Introdução}

O novo coronavírus, SARS-CoV-2 (Severe Acute Respiratory Syndrome Coronavirus 2), causador da doença COVID-

19 (Coronavirus Disease 2019) foi detectado pela primeira vez em 31 de dezembro de 2019 em Wuhan, na China. Diante da rápida disseminação e proporção global alcançada, no dia 12 de março de 2020 a Organização Mundial da Saúde (OMS) declarou a COVID-19 como pandemia (Brito et al., 2020; Ferreira et al., 2020). No Brasil, o primeiro caso da doença foi detectado no dia 
26 de fevereiro de 2020, aproximadamente dois meses após o primeiro caso ter sido notificado na China, e o primeiro óbito em 12 de março de 2020, em São Paulo (Simões \& Silva et al., 2020). Atualmente, o Brasil apresenta mais de 21 milhões de casos da doença e mais de 600 mil mortes, constituindo-se com um dos epicentros da pandemia da COVID-19 (Johns Hopkins Coronavirus Resource Center, 2021).

A transmissão do SARS-CoV-2 ocorre principalmente pelo trato respiratório através de gotículas, secreções ou contato direto. As sintomatologias mais recorrentes incluem febre, diarreia, ageusia, anosmia, tosse, cefaleia, dores musculares, e em casos graves, dispneia ou desconforto respiratório. Alguns fatores de riscos foram evidenciados para a evolução com quadro clínico grave e morte pela doença, que incluem idade avançada, tabagismo e presença de comorbidades, como neoplasias (Brito et al., 2020; Ferreira et al., 2020).

Os pacientes oncológicos podem estar mais susceptíveis as complicações da COVID-19, e consequentemente a um maior risco de morte, devido a imunossupressão comumente causada pelo câncer, e pelos efeitos quimioterápicos, radioterápicos e cirúrgicos do tratamento da doença, que atenuam as respostas imunológicas do paciente (Silva Filho et al., 2020; Araujo et al., 2020). Dessa forma, a Sociedade Brasileira de Oncologia emitiu diretrizes, visando à menor exposição do paciente oncológico à infecção por COVID-19, como diminuição de consultas presenciais e tempo de internação, e adiamento de cirurgias ou quimioterapias, sempre que possível, porém essas medidas podem gerar um forte impacto no tratamento e sobrevida dos pacientes oncológicos (Simões \& Silva et al., 2020).

Além disso, as medidas restritivas acionadas pelos governantes mediante a pandemia, como isolamento social, limitações estabelecidas em consultas na saúde pública e a sobrecarga do Sistema Único de Saúde (SUS) na qual houve a necessidade de priorizar leitos hospitalares e profissionais de saúde voltados ao atendimento de pacientes com COVID-19, pode gerar um decréscimo no diagnóstico de câncer, devido o adiamento ou cancelamento de consultas médicas, a impossibilidade na realização de exames de preventivos de neoplasias e o temor do paciente frente a contaminação hospitalar pelo SARS-CoV-2 (Kuderer et al., 2020).

Apesar da literatura relatar a maior vulnerabilidade de pacientes oncológicos ao risco aumentado de infecção por COVID-19, e seu impacto na sobrevida e diagnóstico da doença (Zhang et al., 2020; Kuderer et al., 2020), trabalhos sobre o tema envolvendo a população brasileira são escassos na literatura. Identificar fatores de risco relacionados aos pacientes oncológicos mediante a pandemia da COVID-19 são relevantes, pois criam bases de dados capazes de justificar a elaboração de protocolos específicos que podem orientar ações a fim de minimizar os impactos negativos na prevenção, no diagnóstico e no tratamento do câncer.

O objetivo do trabalho foi compilar informações na literatura sobre o impacto da pandemia da COVID-19 em pacientes oncológicos brasileiros, em termos de diagnóstico, atendimento e mortalidade.

\section{Metodologia}

O presente estudo trata-se de uma revisão integrativa da literatura, forme descrito por Mendes et al. (2008). Uma pesquisa bibliográfica foi utilizada para identificar estudos sobre o tema: "COVID-19 e Câncer", foram utilizadas três plataformas de pesquisas: Google Scholar, Scientific Electronic Library Online (Scielo) e Pubmed.

A busca aconteceu por três autores de forma independente, utilizando a combinação dos seguintes descritores nos idiomas inglês e português: "COVID-19"; "Câncer"; "Mortalidade"; "Diagnóstico" e "Atendimento". Os critérios de elegibilidade foram artigos originais envolvendo a população brasileira, disponíveis na íntegra de forma gratuita e escritos em inglês e/ou português no período de dezembro de 2019 a junho de 2021. Foram excluídos artigos duplicados, artigos de revisão ou de opinião e aqueles que, após a leitura do título e/ou do resumo, não abordassem os objetivos da nossa pesquisa e/ou a 
população alvo. A triagem da seleção dos artigos que abordavam mais precisamente o tema do trabalho está descrito na Figura 1.

Figura 1. Diagrama de fluxo mostrando o número de registros identificados, excluídos e incluídos da presente revisão.

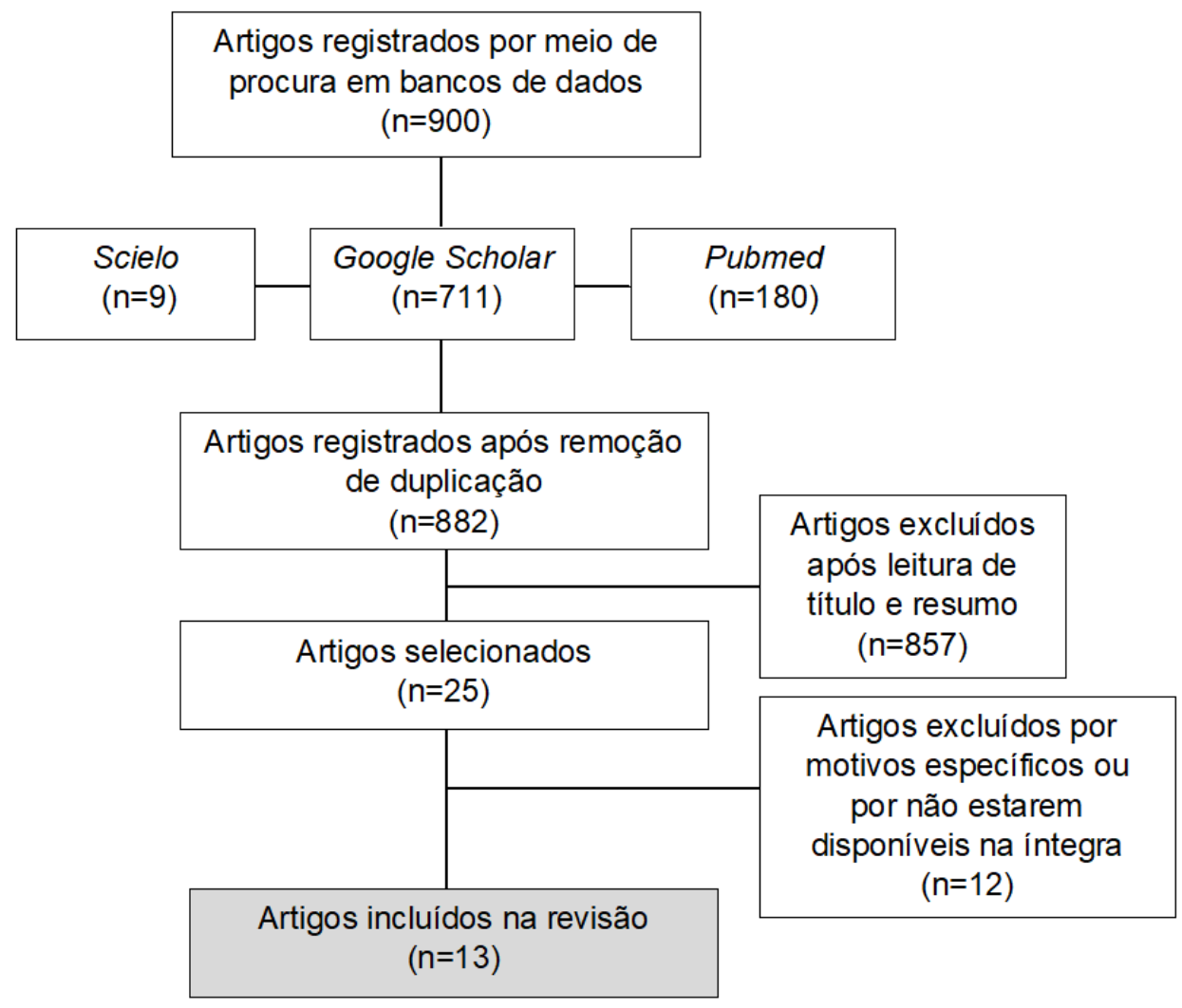

Fonte: Autores.

\section{Resultados}

Após a seleção, um total de 13 estudos foram incluídos na presente revisão. Cinco artigos abordaram mais especificamente dados de diagnóstico de câncer e número de consultas, quatro trabalhos avaliaram mais diretamente dados relacionados ao atendimento e procedimentos médicos como interações e cirurgias e quatro averiguaram o risco de mortalidade em pacientes oncológicos com COVID-19. As populações brasileiras analisadas nos estudos eram de diferentes regiões geográficas, com maior frequência da região sudeste $(33,3 \%)$.

Os estudos envolviam pacientes com diferentes tipos de câncer, com evidência para as neoplasias de cabeça e pescoço, que foram estudadas especificamente em quatro trabalhos. De maneira geral, todos os trabalhos analisados evidenciaram uma redução nos números de diagnóstico de câncer, assim como um déficit nos volumes de consultas, internações e procedimentos cirúrgicos. Também foram evidenciados altas taxas de letalidade para pacientes oncológicos diagnosticados com COVID-19. As regiões Norte e Nordeste foram as mais afetadas. A descrição das publicações selecionas, assim com o resumo dos principais resultados está evidenciado no Quadro 1. 
Quadro 1. Descrição das publicações selecionadas segundo autoria, ano, título, população estudada, tipo de câncer e principais resultados.

\begin{tabular}{|c|c|c|c|c|}
\hline Autor/ano & Título & Região/local & Tipo de Câncer & Principais resultados \\
\hline $\begin{array}{l}\text { Marques et } \\
\text { al., } 2021 .\end{array}$ & $\begin{array}{l}\text { Cancer diagnosis in Brazil in the } \\
\text { COVID-19 era. }\end{array}$ & $\begin{array}{l}\text { Regiões geográficas do } \\
\text { Brasil (Norte, Nordeste, } \\
\text { Sudeste, Sul e Centro- } \\
\text { Oeste). }\end{array}$ & Qualquer neoplasia maligna. & $\begin{array}{l}\text { Redução de } 35,5 \% \text { no número } \\
\text { de casos novos de câncer. }\end{array}$ \\
\hline $\begin{array}{l}\text { Aguiar et } \\
\text { al., } 2021 .\end{array}$ & $\begin{array}{l}\text { Impact of COVID-19 on } \\
\text { colorectal cancer presentation. }\end{array}$ & São Paulo. & Câncer colorretal ou anal. & $\begin{array}{l}\text { Redução de } 46,3 \% \text { nos } \\
\text { diagnósticos. }\end{array}$ \\
\hline $\begin{array}{l}\text { Araujo et } \\
\text { al., } 2020 .\end{array}$ & $\begin{array}{l}\text { Impact of COVID-19 pandemic } \\
\text { on care of oncological patients: } \\
\text { experience of a cancer center in a } \\
\text { Latin American pandemic } \\
\text { epicenter. }\end{array}$ & São Paulo. & Qualquer neoplasia maligna. & $\begin{array}{l}\text { Redução de } 45 \% \text { nas consultas } \\
\text { médicas. } \\
\text { Redução de } 56,2 \% \text { de novas } \\
\text { consultas. } \\
\text { Redução de } 51,2 \% \text { no tempo de } \\
\text { internação e de } 60 \% \text { nas } \\
\text { cirurgias. }\end{array}$ \\
\hline $\begin{array}{l}\text { Abrantes et } \\
\text { al., } 2020 .\end{array}$ & $\begin{array}{l}\text { Oral cancer diagnosis during the } \\
\text { COVID-19 pandemic in an oral } \\
\text { pathology laboratory in Rio de } \\
\text { Janeiro, Brazil. }\end{array}$ & Rio de Janeiro. & Câncer oral. & $\begin{array}{l}\text { Redução de } 51,5 \% \text { no } \\
\text { diagnóstico. } \\
\text { Aumento de } 14,7 \% \text { nas taxas de } \\
\text { malignidade. }\end{array}$ \\
\hline $\begin{array}{l}\text { Da cunha et } \\
\text { al., } 2021 .\end{array}$ & $\begin{array}{l}\text { The impact of the COVID-19 } \\
\text { pandemic on hospitalizations for } \\
\text { oral and oropharyngeal cancer in } \\
\text { Brazil. }\end{array}$ & $\begin{array}{l}\text { Regiões geográficas do } \\
\text { Brasil (Norte, Nordeste, } \\
\text { Sudeste, Sul e Centro- } \\
\text { Oeste). }\end{array}$ & Câncer de boca e orofaringe & $\begin{array}{l}\text { Redução de 49,3\% nas } \\
\text { internações. }\end{array}$ \\
\hline $\begin{array}{l}\text { Martelli et } \\
\text { al., } 2021\end{array}$ & $\begin{array}{l}\text { Impact of the COVID-19 } \\
\text { pandemic in the head and neck } \\
\text { cancer treatment in the Brazil. }\end{array}$ & $\begin{array}{l}\text { Regiões geográficas do } \\
\text { Brasil (Norte, Nordeste, } \\
\text { Sudeste, Sul e Centro- } \\
\text { Oeste). }\end{array}$ & Câncer de cabeça e pescoço. & $\begin{array}{l}\text { Redução de } 35 \% \text { nas cirurgias. } \\
\text { Aumento de } 50,8 \% \text { de } \\
\text { radioterapia e quimioterapia. }\end{array}$ \\
\hline $\begin{array}{l}\text { Araujo-filho } \\
\text { et al., } 2020 .\end{array}$ & $\begin{array}{l}\text { Lung cancer in the era of COVID- } \\
\text { 19: what can we expect? }\end{array}$ & $\begin{array}{l}\text { Regiões geográficas do } \\
\text { Brasil (Norte, Nordeste, } \\
\text { Sudeste, Sul e Centro- } \\
\text { Oeste). }\end{array}$ & Câncer de pulmão. & $\begin{array}{l}\text { Redução de } 7 \% \\
\text { nas internações. } \\
\text { Diminuição de } 13 \% \text { nos } \\
\text { números de biópsias } \\
\text { pulmonares e } 35 \% \text { de } \\
\text { broncoscopias. }\end{array}$ \\
\hline $\begin{array}{l}\text { Fernandes } e t \\
\text { al., } 2021\end{array}$ & $\begin{array}{l}\text { Differences in mortality of cancer } \\
\text { patients with COVID-19 in a } \\
\text { Brazilian cancer center. }\end{array}$ & São Paulo. & $\begin{array}{l}\text { Câncer hematológico, de mama, } \\
\text { órgãos digestivos, próstata, trato } \\
\text { urinário, órgãos respiratórios, } \\
\text { órgãos genitais femininos e sistema } \\
\text { nervoso central. }\end{array}$ & $\begin{array}{l}\text { Mortalidade de } 12,4 \% \text { por } \\
\text { COVID-19 entre os pacientes } \\
\text { com câncer. Maior taxa de risco } \\
\text { para câncer de pulmão e } \\
\text { hematológico. }\end{array}$ \\
\hline $\begin{array}{l}\text { Galino et al., } \\
2021 .\end{array}$ & $\begin{array}{l}\text { Women with cancer and COVID- } \\
\text { 19: an analysis of lethality and } \\
\text { clinical aspects in Pernambuco }\end{array}$ & Pernambuco. & Qualquer neoplasia maligna. & Taxa de letalidade de $72,06 \%$. \\
\hline $\begin{array}{l}\text { Fonseca } \text { et } \\
\text { al., } 2021 .\end{array}$ & $\begin{array}{l}\text { Reduction in the Number of } \\
\text { Procedures and Hospitalizations } \\
\text { and Increase in Cancer Mortality } \\
\text { During the COVID-19 Pandemic } \\
\text { in Brazil. }\end{array}$ & $\begin{array}{l}\text { Regiões geográficas do } \\
\text { Brasil (Norte, Nordeste, } \\
\text { Sudeste, Sul e Centro- } \\
\text { Oeste). }\end{array}$ & Qualquer neoplasia maligna. & $\begin{array}{l}\text { Redução de } 21 \% \text { no número de } \\
\text { internações. Aumento de } 14 \% \\
\text { na taxa de mortalidade. } \\
\text { Diminuição de biópsias }(29 \%) \text {, } \\
\text { colonoscopias }(57 \%) \text {, } \\
\text { mamografias }(55 \%) \text { e cirurgias } \\
\text { oncológicas }(9 \%) \text {. }\end{array}$ \\
\hline $\begin{array}{l}\text { Lima et al., } \\
2021 .\end{array}$ & $\begin{array}{l}\text { COVID-19 cohort on children } \\
\text { with cancer: delay in treatment } \\
\text { and increased frequency of } \\
\text { deaths. }\end{array}$ & Pernambuco (Recife). & $\begin{array}{l}\text { Neoplasias hematológicas e de } \\
\text { tumores sólidos. }\end{array}$ & $\begin{array}{l}\text { Adiamento de } 66,6 \% \text { no } \\
\text { tratamento oncológico. } \\
\text { Mortalidade } 16,6 \% \text { por } \\
\text { COVID- } 19 \text { entre os pacientes } \\
\text { com câncer. }\end{array}$ \\
\hline
\end{tabular}

Fonte: Autores.

\section{Discussão}

Das publicações selecionadas, cinco abordaram mais especificamente dados de diagnóstico de câncer e de consultas. O trabalho desenvolvido por Marques et al. (2021), avaliou o impacto da pandemia da COVID-19 no diagnóstico de câncer nas cinco regiões geográficas do Brasil, comparando a média mensal de novos casos de câncer diagnosticados em 2019 com a do ano de 2020. Os autores observaram um déficit de aproximadamente 35\% no número de novos casos de diagnóstico de câncer 
em todo o território nacional. A região nordeste foi a mais afetada com uma redução de quase $47 \%$. Os autores atribuíram essa redução global ao comprometimento que as consultas médicas tiveram no período pandêmico de 2020. Outro trabalho desenvolvido por Aguiar et al. (2021), relatou uma diminuição de 43,6\% para o ano de 2020, no diagnóstico de câncer colorretal ou anal no Centro de Cirurgia Colorretal do hospital AC Camargo, em São Paulo. Araujo et al. (2020), avaliaram o impacto da pandemia COVID-19 nos volumes de pacientes em um centro de referência no tratamento de câncer, na cidade de São Paulo, comparando o ano de 2019 e de 2020 . Como resultado, eles observaram uma diminuição de $45 \%$ nas consultas médicas totais, incluindo uma redução de 56,2\% de novas consultas, também foi evidenciado uma queda de 51,2\% no tempo de internação e um déficit de 60\% de cirurgias durante o período pandêmico de 2020. O trabalho desenvolvido por Abrantes et al. (2020), compararam o diagnóstico de câncer bucal durante os primeiros seis meses de pandemia da COVID-19 com o período prépandêmico, eles relataram uma redução de 51,5\% nos diagnósticos de câncer oral e uma maior taxa de malignidade dos tumores, 14,7\%, em comparação com o mesmo período de 2019. Nabhen et al. (2020) fizeram um rastreamento na admissão de pacientes em um centro de alta complexidade no tratamento oncológico localizado na região Sul do Brasil durante a pandemia da COVID19 e observaram uma redução estatisticamente significante de $42 \%$ nas consultas médicas de todas as especialidades no ano de 2020, em comparação com o mesmo período pré-pandemia.

Em relação a atendimento e procedimentos médicos como consultas, interações e cirurgias, quatro artigos abordaram mais especificamente esses dados. Da Cunha et al. (2021) documentaram o impacto da pandemia nas taxas de hospitalizações de câncer de oral e orofaringe, usando dados disponíveis no SUS, eles compararam as internações nos primeiros períodos da pandemia com o período pré-pandêmico. Eles observaram uma redução de 49,3\% nas hospitalizações, com menores taxas para a região Norte do país, 60\%. Outro trabalho desenvolvido por Martelli et al. (2021) avaliou o impacto da COVID-19 quanto ao número de cirurgias e procedimentos de radioterapia e quimioterapia em pacientes com câncer de cabeça e pescoço, durante os períodos pré-pandêmico e pandêmico no Brasil. Eles observaram uma redução de $35 \%$ nas cirurgias durante o período pandêmico, sendo a região Norte a mais afetada, com uma redução de 60,7\%. Também foi observado para o mesmo período, um aumento nos procedimentos de radioterapia e quimioterapia nesses pacientes, em torno de $51 \%$, com o maior aumento para a região Nordeste, com $75,1 \%$. Os autores ressaltam mudança nos perfis de protocolos de atendimento de câncer de cabeça e pescoço, uma vez que a ressecção cirúrgica é a base para o tratamento desse tipo de câncer, e essa diminuição nas cirurgias podem comprometer a sobrevida do paciente. Um trabalho desenvolvido por Araujo-Filho et al. (2020), utilizando dados do SUS, avaliou o número de internações hospitalares e procedimentos de diagnósticos intervencionistas, como biópsia e cirurgias, relacionados ao câncer de pulmão, entre março e maio de 2020 em comparação com mesmo período em 2019. Eles relataram uma redução de 7\% nas internações por neoplasia pulmonar, e uma diminuição de $13 \%$ e 35\%, respectivamente, nos números de biópsias pulmonares e de broncoscopias realizadas pelo SUS. Outro trabalho desenvolvimento por Gonçalves et al. (2020), avaliou o impacto da pandemia COVID-19 nos serviços brasileiros de referência em oncologia de cabeça e pescoço comparando o ano 2019 com o ano de 2020, eles relataram uma redução significativa de 39,95\% no número médio de novos casos tratados, além de reduções de consultas $(63,1 \%)$ e cirurgias $(35,1 \%)$ no período pandêmico.

Em relação aos índices de mortalidade de pacientes oncológicos infectados por COVID-19, Fernandes et al. (2020), analisaram a mortalidade por COVID-19 em pacientes em tratamento para diferentes tipos de câncer em um grande hospital de referência, em São Paulo. Dos 411 pacientes analisados, 12,4\% morreram devido ao COVID-19, a mortalidade foi mais frequente em pacientes mais velhos, com média de idade de 63 anos. Maiores taxas de letalidade foram relatadas para câncer de pulmão e o hematológico. O trabalho de Galino et al. (2021) analisou a letalidade de mulheres com neoplasias que apresentaram infecção por SARS-CoV-2 no estado de Pernambuco, através dos registros disponibilizados no SUS. Eles observaram uma alta taxa de letalidade global, de 72,06\% entre as mulheres infectadas. O trabalho de Lima et al. (2021) desenvolvido na região nordeste do Brasil, envolvendo um corte de 48 crianças com neoplasias, atendidas em hospital de referência da região, relatou uma letalidade 
de 16,6\% dos pacientes infectados por COVID-19, as neoplasias hematológicas foram as mais afetadas, além disso foi observado um adiamento de 66,6\% no tratamento oncológico entre os infectados por SARS-CoV-2. O trabalho de Fonseca et al. (2021) analisou o número de procedimentos e hospitalizações durante o período de março a maio de 2020 e compararam com o ano anterior, os resultados do trabalho evidenciaram uma redução de $21 \%$ no número de internações e um aumento de $14 \%$ na taxa de mortalidade intra-hospitalar. Além disso, foi relatado uma diminuição em vários procedimentos como: biópsias (29\%), colonoscopias (57\%), mamografias (55\%) e cirurgias oncológicas (9\%).

\section{Conclusão}

Em conclusão, foi observado que a pandemia impactou seriamente os pacientes oncológicos pela diminuição no número de diagnósticos, consultas, cirurgias e um aumento significativo na taxa de mortalidade hospitalar. Esses achados podem ter agravos negativos no diagnóstico e mortalidade por câncer nos anos seguintes. Dessa forma, estudo futuros deverão ser elaborados para delinear estratégias que minimizem esses impactos por meio de elaboração de protocolos de saúde que assegurem a prevenção, o diagnóstico e a assistência oncológica de qualidade no Brasil.

\section{Referências}

Abrantes, T. C., Bezerra, K. T., Silva, C. N., Costa, L. C., Cabral, M. G., Agostini, M., de Andrade, B., Abrahão, A. C., \& Romañach, M. J. (2020). Oral cancer diagnosis during the COVID-19 pandemic in an oral pathology laboratory in Rio de Janeiro, Brazil. Oral diseases, 10.1111/odi.13669.

Aguiar, S., Riechelmann, R. P., de Mello, C., da Silva, J., Diogenes, I., Andrade, M. S., de Miranda Marques, T., Stevanato, P. R., Bezerra, T. S., Silva, M., Lopes, A., \& Curado, M. P. (2021). Impact of COVID-19 on colorectal cancer presentation. The British journal of surgery, $108(2)$, e81-e82.

Araujo, S., Leal, A., Centrone, A., Teich, V. D., Malheiro, D. T., Cypriano, A. S., Cendoroglo Neto, M., \& Klajner, S. (2020). Impact of COVID-19 pandemic on care of oncological patients: experience of a cancer center in a Latin American pandemic epicenter. Einstein (São Paulo, Brazil), 19 , eAO6282.

Araujo-Filho, J., Normando, P. G., Melo, M., Costa, A. N., \& Terra, R. M. (2020). Lung cancer in the era of COVID-19: what can we expect? Jornal brasileiro de pneumologia: publicação oficial da Sociedade Brasileira de Pneumologia e Tisilogia, 46(6), e20200398.

Brito, S. B.P., Braga, I. O., Cunha, C. C., Palácio, M. A. V., \& Takenami, I. (2020). Pandemia da COVID-19: o maior desafio do século XXI. Vigilância sanitária em debate, 8(2):54-63. 2020.

da Cunha, A. R., Antunes, J., Martins, M. D., Petti, S., \& Hugo, F. N. (2021). The impact of the COVID-19 pandemic on hospitalizations for oral and oropharyngeal cancer in Brazil. Community dentistry and oral epidemiology, 49(3), 211-215.

Fernandes, G. A., Feriani, D., França E Silva, I., Mendonça E Silva, D. R., Arantes, P. E., Canteras, J., da Silva, R. R., \& Curado, M. P. (2021). Differences in mortality of cancer patients with COVID-19 in a Brazilian cancer center. Seminars in oncology, S0093-7754(21)00003-8.

Dantas Ferreira, J., da Silva de Lima, F. C., Pinto Oliveira, J. F., de Camargo Cancela, M., \& de Oliveira Santos, M. (2020). Covid-19 e Câncer: Atualização de Aspectos Epidemiológicos. Revista Brasileira De Cancerologia, 66(TemaAtual), e-1013.

Fonseca, G. A., Normando, P. G., Loureiro, L., Rodrigues, R., Oliveira, V. A., Melo, M., \& Santana, I. A. (2021). Reduction in the Number of Procedures and Hospitalizations and Increase in Cancer Mortality During the COVID-19 Pandemic in Brazil. JCO global oncology, 7, 4-9.

Galindo, R. J. S. C., Andrade, L. B., Sena, G. R., Nogueira, L. R. M., Lima, T. P. F., Lima, J. T. O., \& Orange, F. A. (2021). Women with cancer and COVID19: an analysis of lethality and clinical aspects in Pernambuco. Revista Brasileira de Saúde Materno Infantil (Online), 21(1): 157-165.

Gonçalves, S., Kulcsar, M. A., Matos, L. L., Vartanian, J. G., Carvalho, G. B., Dias, F. L., Farias, T. P., Tosto, E., Santos, C. R., Silva, L. G., Javaroni, A. D., Ramos, G. H., Cavalheiro, B. G., Oliveira, J. C., Cicco, R. D., Mello, L. E., Melo, B., Santos, L. R., Goulart, R. N., \& Kowalski, L. P. (2020). Overview of care for head and neck cancer cases in Brazilian Cancer Centers during the COVID-19 pandemic. Archives of Head and Neck Surgery, 49 : e00322020.

Johns Hopkins Coronavirus Resource Center, 2021. COVID-19 Map. https://coronavirus.jhu.edu/map.html. [Acesso em outubro, 2021].

Kuderer, N. M., Choueiri, T. K., Shah, D. P., Shyr, Y., Rubinstein, S. M., Rivera, D. R., Shete, S., Hsu, C. Y., Desai, A., de Lima Lopes, G., Jr, Grivas, P., Painter, C. A., Peters, S., Thompson, M. A., Bakouny, Z., Batist, G., Bekaii-Saab, T., Bilen, M. A., Bouganim, N., Larroya, M. B., \& et al. COVID-19 and Cancer Consortium (2020). Clinical impact of COVID-19 on patients with cancer (CCC19): a cohort study. Lancet (London, England), 395(10241), 1907-1918.

Lima, A. L. M. A., Borborema, M. C. D., Matos, A. P. R., Oliveira, K. M. M., Mello, M. J. G., \& Lins, M. M. (2021). COVID-19 cohort on children with cancer: delay in treatment and increased frequency of deaths. Revista Brasileira de Saúde Materno Infantil, 21: 299-304.

Marques, N. P., Silveira, D., Marques, N., Martelli, D., Oliveira, E. A., \& Martelli-Júnior, H. (2021). Cancer diagnosis in Brazil in the COVID-19 era. Seminars in oncology, S0093-7754(20)30123-8. 
Research, Society and Development, v. 10, n. 13, e350101321235, 2021

(CC BY 4.0) | ISSN 2525-3409 | DOI: http://dx.doi.org/10.33448/rsd-v10i13.21235

Martelli, A. J., Machado, R. A., Pereira, W. M., Silveira, D., da Cruz Perez, D. E., \& Martelli Júnior, H. (2021). Impact of the COVID-19 pandemic in the head and neck cancer treatment in the Brazil. Oral oncology, 116, 105148.

Mendes, K. D. S., silveira, R. C. C. P., \& Galvão, C. M. (2008). Revisão integrativa: método de pesquisa para a incorporação de evidências na saúde e na enfermagem. Texto \& Contexto Enfermagem, 17, 758-764.

Nabhen, J. J., Ostroski, T., Kozonoe, M. M., Orlandi, D., Tormen, T., \& Ioshii, S. O. (2020). Impact of the COVID-19 pandemic in patient admission to a highcomplexity cancer center in Southern Brasil. Revista da Associação Médica Brasileira (1992), 66(10), 1361-1365.

Silva Filho, P. S. P., Costa, R. E. A. R., Santos, M. B. L. dos, Lima, S. P. N., Silva, L. de A., Freitas, A. S., Silva, J. P., Araújo, L. K. C. de, Cunha, S. H., Araújo, F. W. C., Silva, A. P. A., Alves, D. R. C. F., Mendes, A. M., Leão, C. de A., Kirchesch, C. L., \& Macedo, J. L. (2020). Management of patients with cancer during the COVID-19 pandemic. Research, Society and Development, 9(7), e628974609.

Simões E Silva, A. C., Oliveira, E. A., \& Martelli, H., Jr (2020). Coronavirus Disease Pandemic Is a Real Challenge for Brazil. Frontiers in public health, 8 , 268.

Zhang, L., Zhu, F., Xie, L., Wang, C., Wang, J., Chen, R., Jia, P., Guan, H. Q., Peng, L., Chen, Y., Peng, P., Zhang, P., Chu, Q., Shen, Q., Wang, Y., Xu, S. Y., Zhao, J. P., \& Zhou, M. (2020). Clinical characteristics of COVID-19-infected cancer patients: a retrospective case study in three hospitals within Wuhan, China. Annals of oncology:official journal of the European Society for Medical Oncology, 31(7), 894-901. 